\title{
THE SIMILARITY BETWEEN THE PROPERTIES OF IDEALS IN COMMUTATIVE RINGS AND THE PROPERTIES OF NORMAL SUBGROUPS OF GROUPS
}

\author{
EUGENE SCHENKMAN ${ }^{1}$
}

Introduction. The object of this note is to point out a striking similarity between the properties of the set of normal subgroups of a group with ascending chain condition for normal subgroups and the properties of ideals of a commutative Noetherian ring. In particular it will be seen that a normal subgroup equal to its commutator subgroup is the intersection of pairwise complementary subgroups (analogue of pairwise "relatively prime" ideals). And any normal subgroup modulo which the whole group is semisimple, is the intersection of such subgroups which are irreducible.

The theory is based on making the analogy in the following way: sum of ideals corresponds to the product of normal subgroups; product of ideals corresponds to the commutator of the normal subgroups; and the residual quotient of ideals has an analogue introduced here. The concepts prime ideal, irreducible ideal, and radical of an ideal have analogues for normal subgroups as will be pointed out. For the ideal theory one can consult Northcott [3] or van der Waerden [4].

Preliminaries. In what follows $G$ will denote a group, $E$ the subgroup consisting of the identity element alone, and $A$ and $B$ will denote normal subgroups of $G$. Then $[A, B]$ will denote the subgroup generated by the commutators $[a, b]=a b a^{-1} b^{-1}$ where $a$ is in $A$ and $b$ in $B ; a^{b}$ will denote the conjugate $b a b^{-1}$ of $a . A B$ will denote the group generated by $A$ and $B, A \cap B$ their intersection. $[A, A]=A^{(1)}$ will be the derived group of $A$; and for $n>1 A^{(n)}$ will be the derived group of $A^{(n-1)}$.

Definition. The residual quotient of $A$ by $B$, denoted by $A: B$, will be defined to be the set of elements $g$ of $G$ such that for all $b$ in $B$ $[g, b]$ will be in $A$. It is clear that $A: B$ contains $A$ since $A$ is normal. Modulo $A, A: B$ is the centralizer of $B$; and hence $A: B$ is a normal subgroup of $G$.

Presented to the Society, January 30, 1958; received by the editors December 16, 1957.

1 The author is indebted to Professors Butts, Jacob, Kundert and Wade for an interesting seminar in algebraic geometry; to Professor Koch and the referee for improving the statement of the last proposition; and to the National Science Foundation for financial support. 
Some of the elementary properties of normal subgroups are listed here.

(1) $A B=B A$.

(2) $(A B) C=A(B C)$.

(3) $[A, B]=[B, A]$ is normal in $G$.

(4) $[A, B C]=[A, B][A, C]$.

(5) $A \subseteq A B$.

(6) $[A, B] \subseteq A \cap B$.

(7) $(A \cap B) /[A, B]$ is Abelian.

(8) $[[A B] C] \subseteq[[B, C] A][[C A] B]$.

(9) If $(A \cap B \cap C)$ is its own derived group then $A \cap B \cap C$ $=[[A, B] C]=[[B, C] A]=[[C, A] B]$.

(10) $[(A \cap B), A B] \subseteq[A B]$.

(11) $[(A: B), B] \subseteq A$.

(12) $A \subseteq[A: B]$.

(13) $\left(\cap A_{i}\right): B=\cap\left(A_{i}: B\right)$.

(14) $A:\left(B_{1} B_{2} \cdots B_{n}\right)=A: B_{1} \cap \cdots \cap A: B_{n}$.

(15) $A: B=A: A B$.

Proof. (3) follows from the fact that $[a, b]=[b, a]^{-1}$.

It is clear that the right side of (4) is contained in the left side. On the other hand for $a$ in $A, b$ in $B, c$ in $C$ we have $[a, b c]=[a, b][a, c]^{b}$ and hence the generators of the left side of (4) are contained in the right side. (6) depends on the fact that $[a, b]=a\left(a^{-1}\right)^{b}=b^{a} b^{-1}$. (8) is the theorem on page 64 of [5]. (9) follows from (6) and (7).

The other statements are direct consequences of the definitions.

Radical groups. In what follows we shall assume the maximum condition for normal subgroups; that is, if $A$ is normal in $G$ then $G / A$ has a finite principal series.

Definition. $P$ is an irreducible normal subgroup of $G$ if it is not the intersection of two normal subgroups of $G$ each properly containing $P$.

Definition. If $A$ is normal in $G$ then the radical of $A$, denoted by $\operatorname{Rad} A$, is defined to be the group union of all normal subgroups $K$ of $G$ such that for some $n_{k}, K^{\left(n_{k}\right)}$ is contained in $A$. If $A$ is its own radical then $A$ will be called a radical group or simply a radical.

Remark 1. When $A$ is a normal subgroup of a group $G$ such that $G / A$ has a finite principal series then for some $n$, $(\operatorname{Rad} A)^{(n)}$ is contained in $A$.

Remark 2. It should be observed that $\operatorname{Rad} A$ is the complete inverse image under the natural map from $G$ to $G / A$ of the radical of $C / A$ in the sense of Fitting [1]; and that the radical of Fitting corre- 
sponds to $\operatorname{Rad} E$ as defined above. It is also clear that $G$ modulo a radical is semisimple.

Remark 3. $\operatorname{Rad}[A, B]=\operatorname{Rad}(A \cap B)=\operatorname{Rad} A \cap \operatorname{Rad} B$. The first equality follows from (6) and (7) above. The second one is a direct consequence of the definitions.

Definition. $P$ is a prime normal subgroup of $G$ or $P$ is prime in $G$ if $[A, B] \subseteq P$ implies that either $A \subseteq P$ or $B \subseteq P$.

REMARK 4. $P$ is prime if and only if it is an irreducible radical. For it is clear that if $P$ is prime then it is a radical and it is irreducible. On the other hand if $P$ is an irreducible radical such that $[C, D]$ is contained in $P$; then $[C P, D P]$ is contained in $P$. But $C P \cap D P$ contains $P$ and is Abelian modulo $[C P, D P]$. Since $P$ is a radical $C P \cap D P=P$; since $P$ is irreducible either $C P$ or $D P$ is equal $P$ and hence either $C$ or $D$ is contained in $P$ whence it follows that $P$ is prime.

Remark 5. $P$ is prime if and only if $G / P$ has a unique minimal normal non-Abelian subgroup.

Remark 6. If $P$ is prime then $P: A$ is $G$ or $P$ according as $A$ is or is not contained in $P$. This follows from the definitions with (11) and (12).

REmark 7. Let $\bar{c}$ and $\bar{d}$ denote conjugate classes of elements of $G$ and let $[\bar{c}, \bar{d}]$ and $\bar{c} \bar{d}$ denote the union of all the classes containing $[c, d]$ and $c d$ respectively, for $c$ in $\bar{c}$ and $d$ in $\bar{d}$. Then $P$ is prime in $G$ if and only if when the elements of $[\bar{c}, \bar{d}]$ are in $P$ it follows either that those of $\bar{c}$ or those of $\bar{d}$ are in $P$.

REMARK 8. If $A_{1}, \cdots, A_{n}$ are normal subgroups of $G$ such that $\left[\cdots\left[A_{1}, A_{2}\right] \cdots A_{n}\right]$ is contained in the prime $P$ then for some $i$ between 1 and $n, A_{i}$ is in $P$. If $\bar{c}_{1}, \cdots, \bar{c}_{n}$ are conjugate classes such that the elements of $\left[\cdots\left[\bar{c}_{1} \bar{c}_{2}\right] \cdots \bar{c}_{n}\right]$ are in $P$ then for some $i$ the elements of $\bar{c}_{i}$ are in $P$.

Proposition. If $A$ is a normal subgroup of $G$ contained in the set theoretic union of the primes $P_{1}, \cdots P_{n}$, then $A$ is contained in one of them.

Proof. We shall prove that if $A$ is contained in none of the primes $P_{i}$ for $i=1, \cdots n$ then there is a conjugate class of elements contained in $A$ but not in any of the primes $P_{i}$. For $n=1$ this assertion is clear. If we assume that the assertion is true for $n-1$ primes then for each $i$ there is a class $\bar{c}_{i}$ in $A$ whose elements are not contained in $P_{1}, \cdots P_{i-1}, P_{i+1}, \cdots P_{n}$. It is clear we need only to consider the case where the elements of $\bar{c}_{i}$ are in $P_{i}$ for all $i$ since otherwise the proposition follows. Then let $\left.\left.\bar{c}=\prod_{i=1}^{n}\left[\cdots\left[\bar{c}_{1}, \bar{c}_{2}\right] \cdots \bar{c}_{i-1}\right] \bar{c}_{i+1}\right] \cdots \bar{c}_{n}\right]$. 
The elements of the $j$ th factor of this product do not all belong to $P_{j}$ by the previous remark but the elements of all the other factors are in $P_{j}$. It follows that for each $j$ we can pick an appropriate class as factor in the above product so that the product contains a class in none of the $P_{i}$ as was to be shown.

For further results concerning the case where a group is covered by a union of groups one can be referred to the work of Neumann [2].

We shall need the following lemma.

Lemma. If $A$ is a radical normal subgroup of $G$ such that $G / A$ has a finite principal series, and if $A$ is the intersection of $B$ and $C$ then $A$ is the intersection of any normal subgroups $B^{\prime}$ and $C^{\prime}$ where $B^{\prime} / B$ and $C^{\prime} / C$ are solvable.

Proof. Let $K=B^{\prime} \cap C^{\prime}$. If $K \neq A$ then $K$ contains a subgroup $H$ such that $H / A$ is simple non-Abelian since $A$ is a radical. But $H$ must be in $B$ since otherwise $H B / B$ would not be solvable contrary to the fact that $B^{\prime} / B$ is solvable. Similarly $H$ must be in $C$, and hence $H$ is in $B \cap C$ contrary to the fact that $B \cap C=A$ is properly contained in $H$.

Corollary. If $A$ is a radical such that $A=Q_{1} \cap \cdots \cap Q_{n}$ then $A=\operatorname{Rad} Q_{1} \cap \cdots \cap \operatorname{Rad} Q_{n}$.

If $A=B_{1} \cap \cdots \cap B_{n}$ where no $B_{i}$ can be omitted then we shall say that we have a minimal decomposition of $A$.

TheOREM. Every radical $A$ of a group $G$ such that $G / A$ has a finite principal series has a unique minimal decomposition as an intersection of primes.

Proof. If $A$ is irreducible then it is a prime. Otherwise let $A=B \cap C$ where by the above corollary $B$ and $C$ can be assumed to be radicals. But then by an induction argument on the length of the principal series from $A$ to $G$, we can assume that $B$ and $C$ have decompositions as intersections of primes; hence $A$ has such also.

Now suppose $A$ has two minimal decompositions into primes, $A=P_{i} \cap \cdots \cap P_{n}=R_{1} \cap \cdots \cap R_{n}$; then either $m=n$ and the $P$ 's comprise the same set of groups as the $R$ 's or else it is possible to pick a $P_{i}$ or $R_{j}$ which is contained in no other group from either set. For definiteness suppose it to be $P_{1}$. Then by (13) and Remark 6 $A: P_{1}=P_{1}: P_{1} \cap \cdots \cap P_{n}: P_{1}=G \cap P_{2} \cap \cdots \cap P_{n}=P_{2} \cap \cdots \cap P_{n}$. On the other hand $A: P_{1}=R_{1}: P_{1} \cap \cdots \cap R_{m}: P_{1}=R_{1} \cap \cdots \cap R_{m}$ $=A=P_{1} \cap \cdots \cap P_{n}$ and we have a contradiction since the decompositions were assumed to be minimal. We are forced to conclude that the minimal decomposition is unique. 
The primes determined by the above theorem will be called the primes of $A$.

REMARK 9. If $P$ is a prime containing a radical $A$ with primes $P_{1}, \cdots, P_{n}$ then $P$ contains one of the $P_{i}$. For $\left[\cdots\left[P_{1}, P_{2}\right] \cdots P_{n}\right]$ $\subseteq P_{1} \cap P_{2} \cap \cdots \cap P_{n}=A \subseteq P$ and hence the assertion follows from Remark 8.

REMARK 10. If $A$ is a radical with primes $P_{i}$ for $i=1, \cdots, n$ then $A: B=A$ if and only if $B$ is contained in no $P_{i}$. For $A=P_{1} \cap \cdots \cap P_{n}$ and $\left(P_{1} \cap \cdots \cap P_{n}\right): B=P_{1}: B \cap \cdots \cap P_{n}: B$ by (13); this, by Remark 6, is equal to $P_{1} \cap \cdots \cap P_{n}=A$ if and only if $B$ is contained in no $P_{i}$.

When $A: B=A$ we shall say that $B$ is relatively prime to $A$.

REMARK 11. If both $A$ and $B$ are radicals with minimal decompositions $A=P_{1} \cap \cdots \cap P_{n}, B=R_{1} \cap \cdots \cap R_{m}$ then $A: B=A$ if and only if no $R_{j}$ is contained in a $P_{i}$. This follows from Remarks 9 and 10.

REMARK 12. One might define a primary group $Q$ to be such that if $[A, B] \subseteq Q$ then for some $n$ either $A^{(n)}$ or $B^{(n)}$ is contained in $Q$. It then follows that if $Q$ is a subgroup of a prime $P$ such that $P / Q$ is solvable, then $Q$ is primary. On the other hand the analogy to ideal theory breaks down here, for it is not true that if $Q$ is primary then $\operatorname{Rad} Q$ is a prime as the following example illustrates. Let $H$ be isomorphic to the group of 2 by 2 matrices of determinant one over a field of 5 elements. Then $H$ has a center $Z$ containing an element $z$ of order 2 and $H / Z$ is simple. If $K$ is the direct product of $H$ and $H^{\prime}$ where $H^{\prime}$ is another such group with center element $z^{\prime}$ of order 2, let $G=K /\left(z z^{\prime}\right)$. Then it can be checked that the identity $E$ of $G$ is primary but $\operatorname{Rad} E$ is not prime since it is not irreducible.

\section{Subgroups equal to their derived groups.}

Definition. Two normal subgroups $A$ and $B$ of a group $G$ will be called complementary if $G=A B$. In this case we say each is a complement of the other.

REMARK 13. If $A$ and $B$ are complementary subgroups of a group $G$ equal to its derived group; and if $A^{\prime}$ and $B^{\prime}$ are normal in $G$ such that $A / A^{\prime}$ and $B / B^{\prime}$ are solvable then $A^{\prime}$ and $B^{\prime}$ are complementary. For $A B / A^{\prime} B^{\prime}$ must be solvable and then since $G=G^{(1)}, G=A B=A^{\prime} B^{\prime}$.

REMARK 14. If $G=G^{(1)}$ and if $A$ is a complement of $B$ and also of $C$, then $A$ is also a complement of $[B, C]$ and $B \cap C$. For $A B=G$ and $A C=G$. But because of (4) and (6), $G=G^{(1)}=[A B, A C]$ $\subseteq A[B, C] \subseteq A(B \cap C)$.

REMARK 15. If $A$ is a radical and if $A$ and $B$ are complementary in $G$ then $B$ is relatively prime to $A$. For $B$ cannot be contained in any 
of the primes of $A$ since then $G=A B$ would be also contained in a proper subgroup of $G$. Remark 10 is then applicable.

THEOREM. If $G$ is a group equal to its derived group and if $A=A^{(1)}$ is a normal subgroup of $G$ such that $G / A$ has a finite principal series, then $A$ is the intersection of pairwise complementary subgroups $C_{i}$ such that $C_{i}=C_{i}{ }^{(1)}$ and such that the $C_{i}$ are not intersections of pairwise complementary subgroups.

Proof. If $A$ is not an intersection of pairwise complementary subgroups there is nothing to prove. Otherwise $A$ is the intersection of the complementary groups $D$ and $F$ which because of Remark 13 may be taken equal to their derived groups. The theorem now follows readily by an induction argument on the length of the principal series from $G$ to $A$. For $D$ and $F$ are each intersections of complementary subgroups $D_{i}$ and $F_{i}$ equal to their derived groups and such that the $D_{i}$ and the $F_{i}$ equal to their derived groups and such that the $D_{i}$ and the $F_{i}$ are not intersections of pairwise complementary subgroups; and each $D_{i}$ is complementary to each $F_{i}$ since $D$ is complementary to $F$ and $D_{i}$ contains $D, F_{i}$ contains $F$.

Intersections of cosets. If for $i=1, \cdots, n, C_{i}$ are normal subgroups of $G$ let their intersection be denoted by $C$ and for $j=1, \cdots, n$ let the intersection of all the $C_{i}$ except $C_{j}$ be denoted by $B_{j}$. Then it is clear that modulo $C$ the group generated by the $B_{j}$ is the direct product of the $B_{j}$. In case this direct product is equal to $G$ we have the following situation.

Proposition. Using the notations above, the following statements are equivalent:

(i) $G=B_{1} B_{2} \cdots B_{n}$,

(ii) for every set of elements $a_{1}, \cdots, a_{n}$ in $g, \bigcap_{i=1}^{n} a_{i} C_{i}$ is not vacuous.

Proof. Suppose $G=B_{1} B_{2} \cdots B_{n}$ and suppose the $a_{i} C_{i}$ are given. Then for each $i, G=C_{i} B_{i}$ and hence the coset $a_{i} C_{i}$ can be represented as $b_{i} C_{i}$ where $b_{i}$ is in $B_{i}$. Then the element $b_{1} b_{2} \cdots b_{n}$ is in all the given cosets. Thus, if $G=B_{1} B_{2} \cdots B_{n}$, then $\bigcap_{i=1}^{n} a_{i} C_{i}$ is nonvacuous.

Now suppose that for arbitrary $a_{i}$ in $G, \bigcap_{i=1}^{n} a_{i} C_{i}$ is not vacuous, and let $g$ be arbitrary in $G$. Then $x=g\left(\bmod C_{1}\right)$ and $x=1\left(\bmod C_{i}\right)$ for $i=2, \cdots, n$ have a common solution; that is $g=x c_{i}$ where $x$ is in $B_{1}$. But now an induction argument on the length of the principal series from $G$ to $C$ allows us to conclude that $C_{1}$ is contained in $B_{2} \cdots B_{n}$. It follows that $G=B_{1} B_{2} \cdots B_{n}$ as was to be shown.

REMARK 16. When $\bigcap_{i=1}^{n} a_{i} C_{i}$ contains the element $x$ then it con- 
tains precisely the whole coset $x C$. For the condition that $x$ and $y$ are in $\bigcap_{i=1}^{n} a_{i} C_{i}$ is that $x y^{-1}$ is in $C_{i}$ for each $i$ or that $y$ is in the coset $x C$.

REMARK 17. If the $C_{i}$ are pairwise complementary normal subgroups of a group $G$ such that $G=G^{(1)}$ and such that $G / C$ has a finite principal series, and if $a_{i}$ are arbitrary elements of $G$ then the $n$ congruences $x=a_{i}\left(\bmod C_{i}\right)$ have a common solution and all such common solutions make up precisely a coset of $C$. For in view of the proposition above and Remark 16 it will be enough to show that the $B_{i}$ generate $G$. We do that as follows. There is a non-Abelian factor between $G$ and $C_{2}$ since $G=G^{(1)}$; this factor must occur between $C_{1}$ and $C$ since $C_{1}$ and $C_{2}$ are proper complementary subgroups of $G$. Then if $\bar{C}_{i}=\bigcap_{n=1}^{\infty} C_{i}^{(n)}, \bar{C}_{1}$ must contain $C$; and by Remark $13 \bar{C}_{1}$, $C_{2}, \cdots C_{n}$ are pairwise complementary. Then by Remark $14, G=\bar{C}_{1} B_{1}$ and an induction argument on $n$ allows us to conclude that $\bar{C}_{1}$ is contained in $B_{2} \cdots B_{n}$ whence $G=B_{1} B_{2} \cdots B_{n}$ as was to be shown.

This above is of course, the analogue of the Chinese Remainder Theorem.

\section{BIBLIOGRAPHY}

1. Hans Fitting, Beitrage zur Theorie der Gruppen endlicher Ordnung, Jber. Deutschen Math. Verein vol. 48 (1938) pp. 77-141.

2. B. H. Neumann, Groups covered by finitely many cosets, Publ. Math. Debrecen vol. 3 (1955) pp. 227-242.

3. D. G. Northcott, Ideal theory, London, Cambridge University Press, 1953.

4. B. L. van der Waerden, Moderne algebra, New York, Ungar, 1931.

5. Hans Zassenhaus, Group theory, New York, Chelsea, 1949.

Louisiana State University 